\title{
STOCHASTIC INTEGRAL FOR NON-ADAPTED PROCESSES RELATED TO SUB-FRACTIONAL BROWNIAN MOTION WHEN $H>\frac{1}{2}$
}

\author{
BELHADJ AMEL ${ }^{1, a}$, KANDOUCI ABDELDJEBBAR ${ }^{2, b}$ \\ AND BOUCHENTOUF AMINA ANGELIKA ${ }^{3, c}$
}

\footnotetext{
${ }^{1}$ Laboratory of Stochastic Models, Statistic and Applications, University of Saida-Dr. Moulay Tahar, BP 138, EN-NASR, Saida 20000. Algeria.

${ }^{a}$ E-mail: amel.belhadj@univ-saida.dz

${ }^{2}$ Laboratory of Stochastic Models, Statistic and Applications, University of Saida-Dr Moulay Tahar, BP 138, EN-NASR, Saida 20000. Algeria.

${ }^{b}$ E-mail: abdeldjebbar.kandouci@univ-saida.dz and kandouci1974@yahoo.fr

${ }^{3}$ Mathematics Laboratory, Djillali Liabes University of Sidi Bel Abbes, PB 89, Sidi Bel Abbes 22000, Algeria.

${ }^{c}$ E-mail: bouchentouf_amina@yahoo.fr

\|\|$\|$
}

\section{Abstract}

In this paper, we define the stochastic integral of an anticipating integrand, which is a product of instantly independent process and adapted process, with respect to subfractional Brownian motion based on Ayed and Kuo's approach. This provides a new concept of stochastic integration of non-adapted process. Further, we prove that our anticipating integral is a near-martingale under some conditions.

\section{Introduction}

In recent years, the property of long-range dependence appears in many fields including turbulence, telecommunication, finance and so on. The fractional Brownian motion ( $\mathrm{fBm}$ ) is considered as the most-used process that exhibits this property. The $\mathrm{fBm}\left(B_{t}^{H} ; t \geq 0\right)$ with a Hurst parameter

Received May 06, 2021.

AMS Subject Classification: 60H05, 60G15.

Key words and phrases: Stochastic integral, sub-fractional Brownian motion, non-adapted process, near martingale. 
$H \in(0,1)$ is a centered Gaussian process with covariance

$$
R^{H}(s, t)=\frac{1}{2}\left[t^{2 H}+s^{2 H}+|t-s|^{2 H}\right]
$$

for all $s, t \geq 0, B^{H}$ is a self-similar process with stationary increment and presents a long-range dependence when $H>\frac{1}{2}$.

A more general self-similar Gaussian process considered as an intermediate between standard Brownian motion and fractional Brownian motion has been introduced in Bojdecki et al. [3] and appeared in Dzhaparidze and Van Zanten [5] as the even part of fractional Brownian motion. This process arises from occupation time fluctuations of branching particle systems with Poisson initial condition. The so called sub-fractional Brownian motion $\left(S_{t}^{H} ; t \geq 0\right)$ is a centered Gaussian process with covariance

$$
C^{H}(s, t)=s^{2 H}+t^{2 H}-\frac{1}{2}\left[(t+s)^{2 H}-|t-s|^{2 H}\right],
$$

for all $s, t \geq 0$. For $H=\frac{1}{2}$, both $S^{H}$ and $B^{H}$ coincide with the standard Brownian motion. The sub-fBm preserves many properties of $\mathrm{fBm}$ (selfsimilarity, long-range dependence, and Hölder paths) but not the stationarity of increments. Further, the increments on the intervals $[u, u+r]$ are $[u+r, u+$ $2 r]$ are more weakly correlated than those of $\mathrm{fBm}$. Note that the sub-fBm is neither a Markov processes nor a semimartingale. This fact is considered as a limitation for applying classical stochastic calculus developed by Itô; i.e, to give a sense for $\int_{0}^{t} u_{s} d X_{s}$, the integrator $X$ must be a semimartingale which is not the case for $S^{H}$. Therefore, many of the techniques from stochastic analysis are not available when dealing with $S^{H}$, for this reason various approaches have been proposed. Tudor [22, 24] characterized the Wiener integral's domain with respect to $S^{H}$ for all $H \in(0,1)$. Yan et al. 25] employed the divergence operator to define the stochastic integrals with respect to sub-fBm with $H>\frac{1}{2}$. After that, Shen and Chen [20] defined a stochastic integral with respect to sub-fBm $S^{H}$ with $H<\frac{1}{2}$ that extends the divergence integral from Malliavin calculus. In addition, the pathwise Riemann-Stieltjes integral $\int_{0}^{t} u_{s}(\omega) d S_{s}^{H}(\omega)$ exists for a stochastic process $u$ with $\beta$-Hölder continuous trajectories where $\beta>1-H$ and sub-fBm is Hölder continuous of order $\gamma$ for any $\gamma<H$ on any finite interval (see Young 
26] ). Other limitation of classical Itô integral is the adaptedness of the integrand to the natural filtration of integrator ie, $\int_{0}^{t} u_{s} d X_{s}$ is well defined if and only if $u_{t}$ is adapted to the filtration $\mathcal{F}_{t}=\sigma\left\{X_{t}, t \geq 0\right\}$. Then, the integral is defined like Riemann sums at which the evaluation points are the left endpoints of subintervals. The problem of non-adaptedness of the integrand has been discussed the first time by Itô [11], where he raised the question how to define

$$
\int_{0}^{t} B(1) d B(s), \quad 0 \leq t \leq 1
$$

since $B(1)$ is not $\mathcal{F}_{t}$-adapted. Itô proposed to enlarge the filtration by letting $\mathcal{G}_{t}$ be the field generated by $\mathcal{F}_{t}$ and $B(1)$, ie $\mathcal{G}_{t}=\sigma\left\{\mathcal{F}_{t}, B(1)\right\}, B(1)$ is adapted to $\mathcal{G}_{t}$ and $B_{t}$ is a $\mathcal{G}_{t}$-quasimartingale. Therefore, the integral (3) may be defined as a stochastic integral with respect to quasi-martingale.

There have been several extensions in the literature on anticipating stochastic integration. Let's cite for instance Hitsuda [8], Skorokhod [21]. For more details on the anticipating integrals and their applications, see Buckdahn [4], León and Protter [18], Pardoux and Protter [19], and the reference therein. Ayed and Kuo [1] proposed a new viewpoint for defining this kind of integrals by decomposing the anticipating stochastic integrand into a linear combination of the products of instantly independent and adapted stochastic processes. Then, authors defined a stochastic integral of the product of an adapted process and instantly independent process as a Riemann sum thanks to the classical definition of stochastic integral proved in Kuo [13]. Notice that the evaluation points are the left endpoints of subintervals for the first process and the right endpoints for the second. Motivated by this new approach, many studies have been developed. Itô formula of anticipating integral proved in Kuo and Ayed [1], was generalized to different cases in Kuo et al. [14, 15, 9]. The study of a class of stochastic differential equations with anticipating initial conditions was treated in Khalifa et al. [12]. The Itô isometry based on the new integral for anticipating processes was discussed by Kuo et al. [17]. The near-martingale property of anticipating stochastic integral introduced in Kuo et al. [16] have been recently studied in Hwang et al. [10] and Hibino et al. [7].

Motivated by Ayed and Kuo's [1] idea of integrand decomposition, we define the stochastic integral of an anticipating process which is a product 
of an instantly independent process and an adapted process with respect to $S^{H}$, to be the limit of the corresponding Riemann sum and we show that our stochastic integral admit the near-martingale property.

This paper is arranged as follows: in Section 2, we present some preliminaries on pathwise integral with respect to sub-fBm and integrand's decomposition approach proposed by Kuo and Ayed of anticipating integral. In section 3, we give our result using the above approach by replacing the standard Brownian motion by a sub-fractional Brownian motion and we discuss the near-martingale property of our anticipating integral.

\section{Preliminaries}

We describe some basic facts on pathwise integral with respect to sub$\mathrm{fBm}$.

Let $V_{p}\left(S^{H}, \Delta_{n}\right)$ be the $p$-variation of sub-fBm $S^{H}$ defined by :

$$
V_{p}\left(S^{H}, \Delta_{n}\right)=\sum_{i=1}^{n}\left|S_{t_{i}}^{H}-S_{t_{i-1}}^{H}\right|^{p},
$$

where $\Delta_{n}$ is a partition of the interval $[0, T]$.

We denote by $F V_{p}\left(S^{H}\right)=\lim _{\Delta_{n} \rightarrow 0} V_{p}\left(S^{H}, \Delta_{n}\right)$ the $p$-finite variation of the subfBm and by $B V_{p}\left(S^{H}\right)=\sup _{\Delta_{n}} V_{p}\left(S^{H}, \Delta_{n}\right)$ its $p$-bounded variation.

Proposition 1 (Tudor([23], Proposition 2.3)). For every $p>0$, we have:

- $F V_{p}\left(S^{H}\right)=0, B V_{p}\left(S^{H}\right)<\infty$

- $F V_{p}\left(S^{H}\right)=B V_{p}\left(S^{H}\right)=E\left(|N(0,1)|^{p}\right)$

- $F V_{p}\left(S^{H}\right)=B V_{p}\left(S^{H}\right)=\infty$ if $\quad p>\frac{1}{H}$.

if $\quad p=\frac{1}{H}$.

if $p<\frac{1}{H}$,

where $N(0,1)$ is standard Gaussian random variable.

Thus, $S^{H}$ has a $p$-bounded variation and a $p$-finite variation if $p \geq \frac{1}{H}$.

Young [26] proved that if $f(t)$ and $g(t)$ are continuous paths of finite $p, q$ variation, respectively, where $\frac{1}{p}+\frac{1}{q}>1$, then the integral $\int_{0}^{t} f(s) d g(s)$ may be defined as the corresponding Riemann-Stieltjes sum. Specifically, Feyel and Pradelle [6] showed that if $f$ is $\alpha$-Hölder, and $g$ is $\beta$-Hölder with 
$\alpha+\beta>1$, then the Steiltjes integral $\int_{0}^{T} f(s) d g(s)$ exists and is $\beta$-Hölder. Moreover, for every $0<\varepsilon<\alpha+\beta-1$, we have

$$
\left|\int_{0}^{T} f(s) d g(s)\right| \leq C(\alpha, \beta)\|f\|_{[0, T], \alpha}\|g\|_{[0, T], \beta} T^{1+\varepsilon} .
$$

As claimed above, for every process $\left(u_{t}\right)_{t \in[0, T]}$ with $q$-bounded variations with $q<\frac{1}{1-H}$, the Riemann-Stieltjes integral $\int_{0}^{t} u_{r} d S_{r}^{H}$ is almost surely well defined. Particularly, if $u$ has $\alpha$-Hölder paths for some $\alpha>1-H$, then the Riemann-Stieltjes integral $\int_{0}^{t} u_{r} d S_{r}^{H}$ is well defined and has $\beta$-Hölder paths, for every $\beta<H$.

Then, we can define the stochastic integral for an adapted processes with respect to sub-fBm in pathwise sense for $H>\frac{1}{2}$, and the pathwise integral $\int_{0}^{T} f^{\prime}\left(S_{t}^{H}\right) d S_{t}^{H}$ exists for all $f \in C^{2}(\mathbb{R})$.

Next, present Ayed and Kuo's idea in order to explain anticipating stochastic integral.

From Ayed and Kuo [1], the anticipating integrand $B(1)$ is presented as

$$
B(1)=(B(1)-B(t))+B(t) .
$$

Let us consider the following anticipating integrand

$$
B(1)^{2}=[(B(1)-B(t))]^{2}+2 B(t)[B(1)-B(t)]+B(t)^{2} .
$$

Then, we have to decompose the integrands as $B(1)^{n}, e^{B(1)}$ to get the common property given in the following definition.

Definition 1 (Ayed and Kuo ([1], Definition 2.1 )). A stochastic process $g(t)$ is said to be instantly independent with respect to a filtration $\mathcal{F}_{t}$ if $g(t)$ and $\mathcal{F}_{t}$ are independent for each $t$.

Via the above definition, $B(1)-B(t),[B(1)-B(t)]^{2},[B(1)-B(t)]^{n}$, and $e^{B(1)-B(t)}$ are all instantly independent for $0 \leq t \leq 1$.

Example 1. Let $\mathcal{F}_{t}$ be the underlying filtration of Brownian motion $B(t)$, thus $g(B(1)-B(t))$ is instantly independent of $\mathcal{F}_{t}, t \in[0,1]$, for any real 
measurable function $g(x)$. Nevertheless, $g(B(1)-B(t))$ is adapted to $\mathcal{F}_{t} ; t \geq$ 1 .

Lemma 1 (Ayed and Kuo (2], Lemma 4.1)). If a stochastic process $X(t)$ is both adapted and instantly independent with respect to a filtration $\mathcal{F}_{t}$, then $X(t)$ is a deterministic function.

From this Lemma, we conclude that instantly independent processes are independent of the past and present contrarily to the adapted processes. Thus, we can consider the collection of instantly independent processes as a counterpart of Itô's theory. In addition, we can deduce that many anticipating stochastic processes can be decomposed into sums of the products of an Itô part and a counterpart.

Further, Itô integral measures the integrand using left endpoint for each subinterval. For instantly independent part, if we also use the left endpoint to approximate, we lose its important properties as it has been seen in Example 1. However, if we measure the instantly independent part using right endpoint, its properties will be conserved. This lead to Ayed and Kuo's definition of the new integral.

Definition 2 (Ayed and Kuo ([1], Definition 2.2)). Let $B(t)$ be a Brownian motion, for an adapted stochastic process $f(t)$ with respect to the filtration $\mathcal{F}_{t}$ and an instantly independent stochastic process $g(t)$ with respect to the same filtration, we define the stochastic integral of $f(t) g(t)$ to be the limit:

$$
\int_{0}^{T} f(t) g(t) d B(t)=\lim _{\left\|\Delta_{n}\right\| \rightarrow 0} \sum_{i=1}^{n} f\left(t_{i-1}\right) g\left(t_{i}\right)\left(B\left(t_{i}\right)-B\left(t_{i-1}\right)\right)
$$

provided that the limit in probability exists, where $\Delta_{n}=\left\{0=t_{0}<t_{1}<\right.$ $\left.\cdots<t_{n}=T\right\}$ is the partition of interval $[0, T]$.

Many examples are given in Ayed and Kuo [1, 2].

\section{New Anticipating Integral}

Based on the concept presented above, we give a definition of stochastic integral of the product $f(t) g(t)$ as in Definition 2 by taking the sub-fBm $S^{H}$ as integrator. Formally, we have: 
Definition 3. Let $S^{H}, H>\frac{1}{2}$ a sub-fractional Brownian motion and $\mathcal{F}_{t}$ the $\sigma$-field generated by $\left\{S^{H}(t), t \geq 0\right\}$, for an adapted stochastic process $f(t)$ with respect to the filtration $\mathcal{F}_{t}$ and an instantly independent stochastic process $g(t)$ with respect to the same filtration. We define the stochastic integral of $f(t) g(t)$ to be the limit:

$$
\int_{0}^{T} f(t) g(t) d S^{H}(t)=\lim _{\left\|\Delta_{n}\right\| \rightarrow 0} \sum_{i=1}^{n} f\left(t_{i-1}\right) g\left(t_{i}\right)\left(S^{H}\left(t_{i}\right)-S^{H}\left(t_{i-1}\right)\right)
$$

provided that the limit in probability exists.

It is clear that the anticipating integral (9) is not a $\mathcal{F}_{t}$-martingale to $\mathcal{F}_{t}$ moreover the integrator $S^{H}$ is not a $\mathcal{F}_{t}$-semi-martingale what brings us to verify if it satisfy the near-martingale property presented in Kuo et al. 16.

Definition 4 (Kuo et al. (16], Definition 3.5)). A stochastic process $X_{t}$; $a \leq t \leq b$; with $E\left|X_{t}\right|<\infty$ is called a near-martingale with respect to a filtration $\left\{\mathcal{F}_{t} ; a \leq t \leq b\right\}$ if for any $a \leq s \leq t \leq b$ we have

$$
E\left[X_{t} / \mathcal{F}_{s}\right]=E\left[X_{s} / \mathcal{F}_{s}\right] ; \quad \text { a.s. }
$$

or equivalently

$$
E\left[X_{t}-X_{s} / \mathcal{F}_{s}\right]=0 ; \quad \text { a.s. }
$$

We prove now that, the processes $X_{t}$ and $Y_{t}$ defined by (12) and (17) respectively, are near-martingales for an adapted process $f(t)$ and centered instantly independent process $g(t)$ with respect to the forward filtration

$$
\mathcal{F}_{t}=\sigma\left\{B(s), S^{H}(s) ; 0 \leq s \leq t\right\},
$$

and are near-martingales for a centered adapted process $f(t)$ and instantly independent process $g(t)$ with respect to the backward filtration

$$
\mathcal{F}^{(t)}=\sigma\left\{B(T)-B(s), S^{H}(T)-S^{H}(s) ; 0 \leq s \leq t\right\} .
$$

Theorem 1. Let $\mathcal{F}_{t}$ be a forward filtration and let $f(x)$ and $g(x)$ be continuous functions such that:

$$
\text { 1. } E\left[\int_{0}^{T} f(B(t)) g(B(T)-B(t)) d S^{H}(t)\right]<+\infty \text {, }
$$


2. $E[g(B(T)-B(t))]=0$.

Then

$$
X_{t}=\int_{0}^{t} f(B(s)) g(B(T)-B(s)) d S^{H}(s) ; \quad 0 \leq t \leq T
$$

exists and is a near-martingale with respect to the forward filtration $\mathcal{F}_{t}$.

Proof. We need to verify that $E\left[X_{t}-X_{s} / \mathcal{F}_{s}\right]=0$ for $0 \leq s \leq t$. Notice that

$$
X_{t}-X_{s}=\int_{s}^{t} f(B(u)) g(B(T)-B(u)) d S_{u}^{H} .
$$

Let $\Delta_{n}=\left\{s=t_{0}<t_{1}<\cdots<t_{n-1}<t_{n}=t\right\}$ be a partition of interval $[s, t]$ and $\Delta S_{i}^{H}=S^{H}\left(t_{i}\right)-S^{H}\left(t_{i-1}\right)$, we have:

$$
E\left[X_{t}-X_{s} / \mathcal{F}_{s}\right]=E\left[\int_{s}^{t} f(B(u)) g(B(T)-B(u)) d S^{H}(u) / \mathcal{F}_{s}\right] .
$$

From Definition 3, we have

$$
\begin{aligned}
E\left[X_{t}-X_{s} / \mathcal{F}_{s}\right] & =E\left[\lim _{\left\|\Delta_{n}\right\| \rightarrow 0} \sum_{i=1}^{n} f\left(B\left(t_{i-1}\right)\right) g\left(B(T)-B\left(t_{i}\right)\right) \Delta S_{i}^{H} / \mathcal{F}_{s}\right] \\
& =\lim _{\left\|\Delta_{n}\right\| \rightarrow 0} \sum_{i=1}^{n} E\left[f\left(B\left(t_{i-1}\right)\right) g\left(B(T)-B\left(t_{i}\right)\right) \Delta S_{i}^{H} / \mathcal{F}_{s}\right] .
\end{aligned}
$$

It is sufficient to verify that every component of the last sum is zero. Recall that $f\left(B\left(t_{i-1}\right)\right)$ is $\mathcal{F}_{t_{i-1}}$-measurable and $g\left(B(T)-B\left(t_{i}\right)\right)$ is independent of $\mathcal{F}_{t_{i-1}}$, using the properties of conditional expectation, we obtain:

$$
\begin{aligned}
& E\left[f\left(B\left(t_{i-1}\right)\right) g\left(B(T)-B\left(t_{i}\right)\right) \Delta S_{i}^{H} / \mathcal{F}_{s}\right] \\
& \quad=E\left[E\left[f\left(B\left(t_{i-1}\right)\right) g\left(B(T)-B\left(t_{i}\right)\right) \Delta S_{i}^{H} / \mathcal{F}_{t_{i}}\right] / \mathcal{F}_{s}\right] \\
& \quad=E\left[f\left(B\left(t_{i-1}\right)\right) \Delta S_{i}^{H} E\left[g\left(B(T)-B\left(t_{i}\right)\right) / \mathcal{F}_{t_{i}}\right] / \mathcal{F}_{s}\right] \\
& \quad=E\left[f\left(B\left(t_{i-1}\right)\right) \Delta S_{i}^{H} E\left[g\left(B(T)-B\left(t_{i}\right)\right)\right] / \mathcal{F}_{s}\right] .
\end{aligned}
$$

The independence of Brownian increments and the zero expectation of $g(B(T)-$ 
$\left.B\left(t_{i}\right)\right)$ show that:

$$
\begin{aligned}
& E\left[f\left(B\left(t_{i-1}\right)\right) g\left(B(T)-B\left(t_{i}\right)\right) \Delta S_{i}^{H} / \mathcal{F}_{s}\right] \\
& \quad=E\left[g\left(B(T)-B\left(t_{i}\right)\right)\right] E\left[f\left(B\left(t_{i-1}\right)\right) \Delta S_{i}^{H} / \mathcal{F}_{s}\right] \\
& \quad=0 .
\end{aligned}
$$

Thus, $X_{t}$ is a near-martingale with respect to $\mathcal{F}_{t}$.

Theorem 2. Let $\mathcal{F}_{t}$ be a forward filtration and let $f(x)$ and $g(x)$ be continuous functions such that:

1. $E\left[\int_{0}^{T} f(B(t)) g(B(T)-B(t)) d S^{H}(t)\right]<+\infty$

2. $E[g(B(T)-B(t))]=0$,

then

$$
Y_{t}=\int_{t}^{T} f(B(s)) g(B(T)-B(s)) d S^{H}(s), \quad 0 \leq t \leq T,
$$

exists and is a near-martingale with respect to the forward filtration $\mathcal{F}_{t}$.

Proof. Notice that for $0 \leq s<t \leq T$, we have

$$
Y_{t}-Y_{s}=-\int_{s}^{t} f(B(u)) g(B(T)-B(u)) d S^{H}(u)=-\left(X_{t}-X_{s}\right),
$$

where $X_{t}$ is given in (12). Thus $Y_{t}$ is a near-martingale with respect to $\mathcal{F}_{t}$.

Theorem 3. Let $\mathcal{F}^{(t)}$ be a backward filtration and let $f(x)$ and $g(x)$ be continuous functions such that:

1. $E\left[\int_{0}^{T} f(B(t)) g(B(T)-B(t)) d S^{H}(t)\right]<+\infty$,

2. $E[f(B(t))]=0$,

then

$$
X_{t}=\int_{0}^{t} f(B(s)) g(B(T)-B(s)) d S^{H}(s), \quad 0 \leq t \leq T,
$$

exists and is a near-martingale with respect to the backward filtration $\mathcal{F}^{(t)}$. 
Proof. According to the proof of Theorem 1, we just have to prove that

$$
E\left[f\left(B\left(t_{i-1}\right)\right) g\left(B(T)-B\left(t_{i}\right)\right) \Delta S_{i}^{H} / \mathcal{F}^{(t)}\right]=0,
$$

where $0 \leq s<t \leq T$ and $s=t_{0}<t_{1}<\cdots<t_{n-1}<t_{n}=t$. Notice that

$$
\Delta S_{i}^{H}=\left(S_{T}^{H}-S_{t_{i-1}}^{H}\right)-\left(S_{T}^{H}-S_{t_{i}}^{H}\right) \in \mathcal{F}^{\left(t_{i-1}\right)} .
$$

Next, by the $\mathcal{F}^{\left(t_{i-1}\right)}$ - measurability of $\Delta S_{i}^{H}$ and the conditional expectation properties, we obtain

$$
\begin{aligned}
& E\left[f\left(B\left(t_{i-1}\right)\right) g\left(B(T)-B\left(t_{i}\right)\right) \Delta S_{i}^{H} / \mathcal{F}^{(t)}\right] \\
& \quad=E\left[E\left[f\left(B\left(t_{i-1}\right)\right) g\left(B(T)-B\left(t_{i}\right)\right) \Delta S_{i}^{H} / \mathcal{F}^{\left(t_{i-1}\right)}\right] / \mathcal{F}^{(t)}\right] \\
& \quad=E\left[g\left(B(T)-B\left(t_{i}\right)\right) \Delta S_{i}^{H} E\left[f\left(B\left(t_{i-1}\right)\right) / \mathcal{F}^{\left(t_{i-1}\right)}\right] / \mathcal{F}^{(t)}\right] .
\end{aligned}
$$

Notice that for each $s>t_{i-1}, B(T)-B(s)$ is independent of $\mathcal{F}_{t_{i-1}}$. This implies the independence of the $\sigma$-fields $\mathcal{F}^{\left(t_{i-1}\right)}$ and $\mathcal{F}_{t_{i-1}}$. Since $f\left(B\left(t_{i-1}\right)\right)$ is $\mathcal{F}_{t_{i-1}}$ measurable, it follows that $f\left(B\left(t_{i-1}\right)\right)$ is independent of $\mathcal{F}^{\left(t_{i-1}\right)}$. Thus

$$
\begin{aligned}
& E\left[f\left(B\left(t_{i-1}\right)\right) g\left(B(T)-B\left(t_{i}\right)\right) \Delta S_{i}^{H} / \mathcal{F}^{(t)}\right] \\
& \quad=E\left[g\left(B(T)-B\left(t_{i}\right)\right) \Delta S_{i}^{H} E\left[f\left(B\left(t_{i-1}\right)\right)\right] / \mathcal{F}^{(t)}\right] .
\end{aligned}
$$

Since $E\left[f\left(B\left(t_{i-1}\right)\right)\right]$ is $\mathcal{F}^{(t)}$ - measurable, then

$$
\begin{aligned}
& E\left[f\left(B\left(t_{i-1}\right)\right) g\left(B(T)-B\left(t_{i}\right)\right) \Delta S_{i}^{H} / \mathcal{F}^{(t)}\right] \\
& \quad=E\left[f\left(B\left(t_{i-1}\right)\right)\right] E\left[g\left(B(T)-B\left(t_{i}\right)\right) \Delta S_{i}^{H} / \mathcal{F}^{(t)}\right] \\
& \quad=0 .
\end{aligned}
$$

Theorem 4. Let $\mathcal{F}^{(t)}$ be a backward filtration and let $f(x)$ and $g(x)$ be continuous functions such that: 
1. $E\left[\int_{0}^{T} f(B(t)) g(B(T)-B(t)) d S^{H}(t)\right]<+\infty$,

2. $E[f(B(t))]=0$,

then

$$
Y_{t}=\int_{t}^{T} f(B(s)) g(B(T)-B(s)) d S^{H}(s), \quad 0 \leq t \leq T,
$$

exists and is a near-martingale with respect to the backward filtration $\mathcal{F}^{(t)}$.

Proof. From Theorem 3, we have $Y_{t}-Y_{s}=-\left(X_{t}-X_{s}\right)$. Consequently, the proof is completed.

\section{Acknowledgments}

We acknowledge funding from ATRST (Algeria) for the research results approved in this work.

\section{References}

1. W. Ayed and H. H. Kuo, An extension of the Itô integral, Communications on Stochastic Analysis, 92 (2008), 323-333, .

2. W. Ayed and H. H. Kuo, An extension of the itô integral: toward a general theory of stochastic integration, Theory of Stochastic Processes, 16 (2010), 17-28.

3. T. Bojdecki, L. Gorostiza and A. Talarczyk, Sub-fractional brownian motion and its relation to occupation times, Statistics $\& 3$ Probability Letters, 69 (2004), 405-419.

4. R. Buckdahn, Anticipative girsanov transformations, Probability Theory and Related Fields, 89 (1991), 211-238.

5. K. Dzhaparidze and J. V. Zanten, A series expansion of fractional brownian motion, Probability Theory and Related Fields, 130 (2002), 39-55.

6. D. Feyel and A. Pradelle, On fractional brownian processes, Potential Analysis, 10 (1999), 273-288.

7. S. Hibino, H. H. Kuo and K. Saitô, A stochastic integral by a near-martingale, Communications on Stochastic Analysis, 12 (2018), 197-213.

8. M. Hitsuda, Formula for brownian partial derivatives, Second Japan USSR Symposium on Probability Theory, Kyoto, 111-114, 1972.

9. C. R. Hwang, H. H. Kuo, K. Saitô and J. Zhai, A general itô formula for adapted and instantly independent stochastic processes, Communications on Stochastic Analysis, 10 (2016), 341-362. 
10. C. R. Hwang, H. H. Kuo, K. Saitô and J. Zhai, Near-martingale property of anticipating stochastic integration, Communications on Stochastic Analysis, 11 (2017), 491-504.

11. K. Itô, Extension of stochastic integrals, Proc. Internat. Symp. Stoch. Diff. Equations, Res. Inst. Mathemat. Sci., Kyoto Univ., Kyoto, 1978.

12. N. Khalifa and H. H. Kuo, Linear stochastic differential equations with anticipating initial conditions, Communications on Stochastic Analysis, 7 (2013), 245-253.

13. H. H. Kuo, Introduction to Stochastic Integration, Springer, New York, 2006.

14. H. H. Kuo, Y. Peng and B. Szozda, Itô formula and girsanov theorem for anticipating stochastic integrals, Communications on Stochastic Analysis, 7 (2013), 441-458.

15. H. H. Kuo, Y. Peng and B. Szozda, Generalization of the anticipative girsanov theorem, Communications on Stochastic Analysis, 7 (2013), 573-589.

16. H. H. Kuo, A. Sae-Tang and B. Szozda, A stochastic integral for adapted and instantly independent stochastic processes, Stochastic Processes, Finance and Control: A Festschrift in Honor of Robert J Elliott (S. N. Cohen, D. Madan, T. K. Siu and H. Yang, eds.), World Scientific, 53-71, 2012.

17. H. H. Kuo, A.Sae-Tang and B. Szozda, An isometry formula for a new stochastic integral, Quantum Probability and Related Topics (L. Accardi and F. Fagnola, eds.), World Scientific, 222-232, 2013.

18. J. A. León and P. Protter, Some formulas for anticipative Girsanov transformations, CRC Press, 1994.

19. E. Pardoux and P. Protter, A two-sided stochastic integral and its calculus, Probability Theory and Related Fields, 76 (1987), 15-49.

20. G. Shen and C. Chen, Stochastic integration with respect to the sub-fractional brownian motion with $H \in(0,1 / 2)$, Statistics $\&$ Probability Letters, 82 (2012), 240-251.

21. A. Skorokhod, On a generalization of a stochastic integral, Theory of Probability \& Its Applications, 20 (1976), 219-233.

22. C. Tudor, Inner product spaces of integrands associated to subfractional brownian motion, Statistics \&f Probability Letters, 78 (2008), 2201-2209.

23. C. Tudor, Some aspects of stochastic calculus for the sub-fractional brownian motion, Ann. Univ. Bucuresti, Mathematica, (2008), 199-230.

24. C. Tudor, On the wiener integral with respect to a sub-fractional brownian motion on an interval, Journal of Mathematical Analysis and Applications, 351 (2009), 456-468.

25. L. Yan, G. Shen and K. H, Itô's formula for a sub-fractional brownian motion, Communications on Stochastic Analysis, 5 (2011), 135-159.

26. L. C. Young, An inequality of the hölder type, connected with stieltjes integration, Acta Mathematica, 67(1936), 251-282. 